\title{
Predicting successful outcomes of complete denture therapy
}

\author{
Abstracted from \\ Critchlow SB, Ellis JS. \\ Prognostic indicators for conventional complete denture therapy: \\ a review of the literature. I Dent 2010; 38: 2-9 \\ Address for correspondence: Janice S Ellis, Department of Restorative Dentistry, \\ School of Dental Sciences, Framlington Place, Newcastle University, \\ Newcastle upon Tyne, UK. E-mail: j.s.ellis@ncl.ac.uk
}

\section{Question: What are the indicators for success in prosthodontic treatment?}

\author{
Data sources Ovid databases searched for relevant material for \\ the review. \\ Study selection Studies were limited to the English language and all \\ identified articles were included. \\ Data extraction and synthesis A narrative review was conducted. \\ Results Three RCT were identified that related directly to conventional \\ dentures. Nineteen clinical experimental studies and seven \\ nonexperimental clinical studies were therefore also included because \\ of the lack of higher ranking studies in this area. All studies that were \\ identified have been included within this review. \\ Conclusions There remains a paucity of research in this area. From \\ the best available data, construction of technically correct dentures, \\ a well-formed mandibular ridge and accuracy of jaw relations are \\ positive indicators for success. Patient neuroticism and a poorly formed \\ mandibular ridge are negative indicators for success. Other prognostic \\ indicators have not been shown to be of significant value. There exists a \\ minority of patients who will never adapt to any conventional complete \\ denture. This problem is more acute in the mandible than the maxilla. \\ There is need for further research in this area.
}

\section{Commentary}

Despite its inherent limitations, this literature review was good enough to show that the scientific basis that dentists use to judge the success or failure of conventional complete denture therapy is weak. The review suggested a number of indicators to predict the success or failure of conventional complete denture therapy, namely: patient age and demographics, psychological factors and personality traits, expectations and attitudes, residual ridge form and anatomy, method of construction, quality of denture and changes over time, and aesthetics. Only a few indicators showed some kind of an association. A positive association would lead to a more successful treatment whereas a negative association would lead to a less successful treatment.

Stating some of what is known anecdotally or through practice, the review concluded that patients with neuroticism would be most likely to be be dissatisfied with their complete dentures. The lower ridge form and quality was found to be more indicative of failure than the maxillary ridge. Past experience, and expectations and attitudes of the patient were not indicative of failure or success. The review also concluded that the literature was equivocal about the methods of construction, duration of use, and aesthetics. Interestingly, however, the review concluded that the age of the patient cannot be considered an indicator for a successful therapy.

It is apparent that this narrative review was done with the basic tenets of evidence-based dentistry in mind. Nonetheless, because of the intrinsic shortcomings of narrative reviews, that is, the absence of a specific research question, lack of predetermined selection criteria and, above all, lack of rigorous assessment of the methodology of the selected studies, the conclusions of the review cannot be considered evidence-based.

Thus arises the legitimate question: how do we benefit from reading this literature review? The answer would be, in a nutshell, that this review showed that after decades of providing patients with conventional complete dentures, the dental profession still lacks good quality studies to show what factors can really lead to a satisfied conventional complete denture patient. 